\title{
The Supernova Working Group
}

\author{
Chair: Wolfgang Hillebrandt \\ Co-Chair: Brian Schmidt
}

\begin{abstract}
The Supernova Working Group (SNWG) was re-established at the General Assembly in Sidney on July 21, 2003, sponsored by Commissions 28 (Galaxies) and 47 (Cosmology). Here we report on some of its activities since 2003 .
\end{abstract}

Keywords. Supernovae

\section{Introduction}

The first SNWG was founded by Fritz Zwicky to coordinate and provide uniform standards for photographic supernova searches in the era of the Palomar 48" and other Schmidt telescopes. At the time, there was no IAU Commission on High Energy Astrophysics, so Galaxies (28) was the natural host. The WG died with him in 1974 (and his personal record of more than 100 SN discoveries held for many years). Commission 28 re-established an SNWG (with Virginia Trimble as chair) at the 1982 Patras General Assembly, largely because of concerns that the few events then being discovered (mostly serendipitously) were not receiving enough follow-up observations to be very information. The WG distributed a document called "in case of supernova, break glass" and perhaps had some small part in preparing the astronomical community to take full advantage of SN 1987A. That even, in turn, made astronomy so supernova-conscious that the WG hardly seemed needed, and it died a natural death at the 1991 General Assembly.

The wish to request permission of the IAU Executive Committee to re-establish a SuperNova Working Group (SNWG) arose from the large number of discoveries (up to several 100 per year), some at very large distances, being made by more than half a dozen teams in the past few years. This was beginning to lead to competition for telescope time for follow-up work which would be unnecessary if the scientists involved had a platform for information exchange. LOTOSS was taking up speed. The Nearby Supernova Factory, the Carnegie Supernova Project, ESSENCE, and other projects to discover and observe SNe Ia at low and intermediate redshifts had left the planning stage in Europe, Japan, and the USA and were thought also to benefit from better coordination. Also, special space probes SNAP were being discussed aiming at the discovery of hundreds of supernovae at all redshifts alone. Finally, considerable progress in modeling the various types of events made it desirable also to have theoretical results, including explosion models, synthetic light curves, and predicted spectra, easily accessible.

Therefore, the aim of the new SNWG was (a) to take care of coordinating observational activities, including searches (e.g. SCP, High-z Team, GOODS, ESSENCE, LOTOSS, CSP, RTN/ESC, etc.), (b) to advocate suitable follow-ups, (c) to establish ways to archive $\mathrm{SN}$ data and to make them available to the community, (d) to edit an electronic newsletter complementary to the IAU Circulars (the GCN network for gamma ray bursters being a possible model), and (e) to be the interface with the coming virtual observatory initiatives (AVO, NVO, ASTRO-Grid, GAVO...) for data storage and handling. 


\section{Tasks of the Supernova Working Group}

At an inaugural meeting (which was part of the Division VIII/Commission 28 business session in Sydney) the objectives of the WG were discussed and approved. In particular it was agreed

- to coordinate the nearby searches and the follow-up observations,

- to launch a community effort for a Treasury or Large HST programme to observe type Ia supernovae in the UV,

- to investigate the possibility of having a robotic (2m-class) telescope devoted to supernova observations,

- to find ways to save supernova data in some form of database, freely accessible to the community.

In addition, it was suggested that the SNWG should take an active role in the discovery and observation reports of supernovae, presently done via IAU Circulars, which involves some unwanted delay. Here the problem is, however, that rapid communication in an 'unfiltered' way increases the risk of 'false alarms'. A second problem is going to be how to cope with 100's of supernovae per year. A possible solution could be that IAU Circulars could continue to be the place for 'confirmed' discoveries, preceded by rapid communications in a web-based platform.

Finally, Wolfgang Hillebrandt (Garching, Germany) and Brian Schmidt (Mt. Stromolo, Australia) were elected as Co-Chairs of the new SNWG.

\section{Activities of the Supernova Working Group since 2003}

Here we summarize briefly some of the SNWG after it was re-established in 2003.

\subsection{Coordinated observations of nearby type Ia supernovae}

One of the major activities of the SNWG was to initiate an HST proposal to observe type Ia supernovae in the UV. In the end, 95 scientists signed-up for a large community proposal with the goal to improve substantially our physical knowledge of SNe Ia and their calibration as cosmological distance probes. The team consisted of a major fraction of the leading researchers (both observational and theoretical) on SNe Ia and their cosmological uses. The approach was twofold: It was planned to study the brightest, closest SNe Ia to make maximum progress in advancing our understanding of the physics of SNe Ia, and to utilize Hubble-flow SNe Ia, where accurate relative luminosities can be determined, to search for and to constrain subtle effects that could compromise their utility for precision cosmology and to produce template light curves in the UV.

The proposal was successful, and was awarded with 152 orbits in Cycle 13, with the prospect of an extension into Cycle 14. Unfortunately on August 3, 2004, just after the program had started STIS (the Space Telescope Imaging Spectrograph) stopped science operations and was put in "safe" mode from which it was not recovered. After lengthy discussions it was decided to go on with the planned observations with the ACS grism despite the failure of STIS. In the following year only two SNe were close enough and were detected early enough to make an attempt to obtain early UV spectra (SN 2004dt and SN 2005cf). At present the data are being analyzed and will become available to the community soon. However, their quality was insufficient to reach all scientific goals and, therefore, the program was not approved for Cycle 14 and was stopped.

In any case, the enormous and unique coordinated effort that went into the observations of these two supernovae makes them the best-covered SNe Ia ever. This includes photometry and spectroscopy in all wave bands, with many telescopes and instruments, 
on ground and in space, and at very many epochs, from about two weeks before maximum light all the way into the late nebular phase. These unprecedented data and their interpretation will shed new light on the physics of the explosions.

In addition, closer contacts between groups searching for nearby supernovae (including amateurs) and those doing the follow-ups were established. As a result, in the past years the number of very nearby SNe Ia's discovered at least 10 days before maximum light and followed closely from then on has more than doubled. The Carnegie Supernova Project, aiming at type Ia and type II supernovae at redshifts below about 0.07 has recently started its operation and will increase much further.

\subsection{Surveys and follow-ups of supernovae in the Hubble flow and at high redshifts}

The Nearby Supernova Factory, an international collaboration between several groups in the United States and France, is designed to address a wide range of supernova issues using detailed observations of low-redshift SN. During the first year of its prototype operation it discovered already 34 supernovae of all types. In June 2004 the SuperNova Integral Field Spectrometer (SNIFS) "saw" its first supernova and is meanwhile fully operational towards its goal is to find and study over 300 nearby type Ia supernovae.

ESSENCE (Equation of State through SupErNova Cosmology Experiment) and the Canada-France-Hawaii Telescope Supernova Legacy Survey are five-year projects which collect 200 and 700 distant $(0.2<\mathrm{z}<0.8)$ Type Ia Supernovae, respectively. They have finished three years of the project now and first results are appearing.

The CFHT Legacy Survey officially started on May 30th 2003, and from June 30, 2006, the data from the first year of observations will become public, the final goal being to establish a photometric data base for around 1000 supernovae in five years.

The supernova results are typically compared to models with an equation of state of the unknown component (dark energy). The supernova data are currently not sufficient to constrain any time dependence. Therefore new projects are presently discussed, and it will take the next generation supernova projects (Dark Energy Camera, PanSTARRS, LSST, JDEM/SNAP or DUNE) to address the equation-of-state question.

The SNWG is taking an active part in several of these projects.

\subsection{Conferences and meetings}

Since its revival the SNWG took an active role in organizing several supernova conferences and workshops.

A workshop on "Thermonuclear Supernovae and Cosmology" was held at Trento, Italy, in September 2003, and a workshop on "Ground-based Supernova Surveys" took place at the University of Chicago in November, 2003, to name a few.

In June, 2004, a major conference was held at the Aula Magna in the Palazzo del Bo in Padua, Italy. The conference, attended by more than 100 researchers from all over the world, was scheduled close to the 400th anniversary of Kepler's supernova SN 1604. Therefore the program included several talks on the history of supernova observations in a session named "New Stars for a New Astronomy", but also the modern aspects of supernova searches, observing programs, and modeling of the events. The meeting was a great success, and the City of Padua decided to devote a prize for research in astronomy which was awarded for the first time in 2005 to Saul Perlmutter (Berkeley) and Brian Schmidt (Mt. Stromolo) for their discovery of the accelerated Universe by using SNe Ia as distance indicators.

In summer 2004, three months of the Institute for Nuclear Theory at Seattle's program was devoted to supernovae and gamma ray burst. The topics covered (also by program workshops) included open issues in understanding core collapse supernovae, supernova 
theory and nucleosynthesis, the supernova - gamma ray burst connection, and type Ia supernovae and cosmology. This program gave young astronomers a chance to meet the experts in their field of research in the relaxed atmosphere of the INT.

Finally, the SNWG will organize a Joint Discussion on "Supernovae: One Millennium after 1006" at the IAU General Assembly in Prague.

\section{Future activities: Supernova data bases and related issues}

A PERL-based platform was developed to allow astronomers to enter suspected supernova discoveries into a data base that will alert registered subscribers directly and automatically. This platform is presently been tested with real data and, in case of success will be made available to the community. In preparation of its release, a web-page will be set up shortly.

Also, supernova data (raw data and reduced data) obtained during some of the European Supernova Collaboration's (ESC) recent observing campaigns of very nearby SNe Ia were given to the German Virtual Observatory to be used as a test bed for tool development. In the end, this data base should not only contain observations but also theory information. It is planned to set up a server at the Max-Planck-Institute for Astrophysics at Garching, Germany.

Wolfgang Hillebrandt Chairperson of the Working Group 